\title{
Highly sensitive detection of CTLA-4-positive T-cell subgroups based on nanobody and fluorescent carbon quantum dots
}

\author{
WU WANG ${ }^{*}$, XIAOQIONG HOU* ${ }^{*}$ XIAOMEI YANG, AIQUN LIU, ZHUORAN TANG, \\ FENGZHEN MO, SHIHUA YIN and XIAOLING LU \\ School of Basic Medicine/International Nanobody Research Center of Guangxi, \\ Guangxi Medical University, Nanning, Guangxi 530021, P.R. China
}

Received June 9, 2018; Accepted February 21, 2019

DOI: $10.3892 / \mathrm{ol} .2019 .10320$

\begin{abstract}
The detection of cytotoxic T-lymphocyte antigen-4-positive (CTLA-4 ${ }^{+}$) T-cell subgroups in peripheral blood samples and tumor tissues is of great significance. In the present study, a rapid, succinct and efficient method was designed for the detection of CTLA $-4^{+}$human T cells using a CTLA-4-specific nanobody-fluorescent carbon quantum dots complex (QDs-Nb36). QDs-Nb36 was used for high sensitivity detection of CTLA- $4^{+} \mathrm{T}$ cells by flow cytometry or immumofluorescent staining. The present study demonstrated that the novel technique was more specific and effective in the detection of CTLA- $4^{+}$T-cell ratio in the peripheral blood and tumor tissues compared with a traditional monoclonal antibody approach. Furthermore, no significant toxicity was identified in vitro and in vivo, thus suggesting that the method may have broad applications for the detection of certain lowly expressed targets.
\end{abstract}

\section{Introduction}

Cytotoxic T-lymphocyte antigen-4 (CTLA-4) is a characteristic inhibitory regulator expressed on the membrane of activated $\mathrm{T}$ cells, which serves as an immunoregulatory role and is responsible for T-cell expansion by suppressing the action of cluster of differentiation (CD)28 (1). CTLA-4 may terminate activation of the T-cell response by downregulating ligand expression levels and emitting inhibitory signals $(2,3)$. A previous study confirmed that the CTLA-4 signaling pathway is a strong inducer in regulating the function of regulatory

Correspondence to: Professor Xiaoling Lu, School of Basic Medicine/International Nanobody Research Center of Guangxi, Guangxi Medical University, 22 Shuangyong Road, Nanning, Guangxi 530021, P.R. China

E-mail: luwuliu@163.com

*Contributed equally

Key words: nanobody, fluorescent carbon quantum dots, cytotoxic T-lymphocyte antigen- $4, T$ cell
T cells (Tregs), which serve a key role in immunity and tolerance. In humans, the major cell type expressing CTLA-4 are Tregs (4), which are a potent controller of the immune response. CTLA-4 is constitutively expressed in Tregs and upregulated in activated conventional $\mathrm{T}$ cells, which are classified as suppressor T-lymphocyte subgroups (5). Previous studies have suggested that CTLA-4 ${ }^{+}$T-cell subgroups are disrupted when the human immune system is weakened, the occurrence of CTLA-4 $4^{+}$T cells is imbalanced at tumor sites and in the peripheral blood of patients with cancer, and this may be associated with clinical diagnosis and prognosis (6-8). Consequently, the detection and analysis of this specific CTLA-4 $4^{+}$lymphocyte subgroup serves a significant role.

Multiple methods have been employed to detect CTLA- $4^{+}$ $\mathrm{T}$ cells, and fluorescent-conjugated monoclonal antibodies (mAbs) are the most commonly utilized. Due to the low proportion of these CTLA- $4^{+}$T-cell subgroups in the peripheral blood and tumor tissues, and interference from the tumor microenvironment, their detection is difficult and costly. Therefore, a simpler and more sensitive assay is urgently required to monitor CTLA- $4^{+}$T-cell subgroups. Nanobodies (Nbs) are a special type of single-domain antibodies consisting of different regions of heavy chain antibodies, which are present in the blood of camels. Compared with conventional antibodies, Nbs possess several inherent characteristics, including high specificity, high physiochemical stability, lack of immunogenicity, high yield and low cost, which make them suitable for immune-targeted diagnosis and treatment of cancer (9). In our previous study, a CTLA-4-specific Nb (Nb36) was screened (10). Nb36 may recognize unique CTLA-4 epitopes and effectively bind with CTLA- $4^{+}$T cells. Due to their relatively complex structures and large molecular masses, traditional antibodies cannot effectively penetrate the barrier of blood vessels and tumor tissues to successfully bind the tumor antigen. Nbs possess a molecular mass of $15 \mathrm{kDa}$, no chemical hydrophobicity, potent heat resistance and high resistance to acids and alkalis (11). Therefore, it has been hypothesized that Nbs may be used in the detection of certain targets with reduced expression. Recently, nanometer-sized optic-functional semiconductors, particularly fluorescent carbon quantum dots (QDs), have attracted widespread attention, and have been utilized as photographic developers in diagnostic instruments $(12,13)$. QDs, 2.5-6 nm in size, have 
a wider excitation spectrum and narrower emission spectrum than traditional organic fluorescent dyes (14); therefore, QDs have been widely used as optical imaging probes. Furthermore, QDs exhibit excellent biocompatibility, non-toxicity and high light stability, and are convenient to modify (15), which are useful in detecting biomarkers or cell surface markers, when combined with a targeting probe (16). Due to their specific optical characteristics, the use of QDs leads to high sensitivity in multiple techniques, including immunohistochemical staining, flow cytometry, fluorescence in situ hybridization and biochips, widening their application range in clinical practice.

To the best of our knowledge, the construction of detection tools based on QDs combined with Nbs has yet to be reported. In the present study, a novel complex based on QDs in combination with Nbs was synthesized to detect CTLA-4 ${ }^{+}$ T cells (QDs-Nb36; Fig. 1). The efficacy of the novel complex and conventional mAbs in target detection was statistically compared.

\section{Materials and methods}

Cells and animals. Peripheral blood T cells were isolated from the whole blood samples of healthy donors (2 males and 2 females, aged between 23 and 33, with normal blood routine, normal liver and kidney function, and no serious diseases such as malignant tumors) using Ficoll-Hypaque density-gradient centrifugation $\left(20 \mathrm{~min}, 24^{\circ} \mathrm{C}, 800 \mathrm{x} \mathrm{g}\right)$. Written informed consent was obtained from all donors. The study procedures were approved by The Local Ethics Committee of Guangxi Medical University (Nanning, China). The 293-cell line was purchased from American Type Culture Collection (Manassas, VA, USA).

A total of 60 female C57BL/6 mice, aged 4-6 weeks, between 13 and $18 \mathrm{~g}$, were purchased from Guangxi Laboratory Animal Center (Guangxi, China) and raised in laminar flow cabinets in a specific pathogen-free environment (temperature, $23 \pm 1^{\circ} \mathrm{C}$; humidity, $50 \pm 10 \%$; 12 -h light/dark cycle starting at 7:00 a.m.; with free access to food and water). All protocols were approved by the Animal Ethics Committee of Guangxi Medical University (Guangxi, China).

Patients. A total of five patients (aged between 35 and 63 years old; 3 males and 2 females) admitted to The First Affiliated Hospital Guangxi Medical University (Guangxi, China) between October 2016 and January 2018, and eligible for surgical resection of hepatocellular carcinoma were enrolled in the present study. Written informed consent was obtained from all patients. Paired tumor tissue and adjacent mucosa ( $\geq 3 \mathrm{~cm}$ from the tumor) samples were obtained after hepatectomy and stored in sterile PBS solution at room temperature. The study procedures were approved by The Local Ethics Committee of Guangxi Medical University.

Reagents. QDs were purchased from Beijing Zhongke Wu Yuan Biotechnology Co., Ltd. (Beijing, China). Ficoll-Paque ${ }^{\mathrm{TM}}$ Plus was purchased from GE Healthcare Life Sciences (Shanghai, China). 1-ethyl-3-(3-dimethylaminopropyl) carbodiimide hydrochloride (EDC) and N-hydroxysulfosuccinimide sodium salt (sulfo-NHS) were purchased from Sigma-Aldrich (Merck KGaA, Darmstadt, Germany). The nuclear dye DAPI was purchased from Thermo Fisher Scientific, Inc. (Waltham, MA, USA). Anti-CTLA-4 mAb (cat. no. Ab7222, $100 \mu \mathrm{g} / 100 \mu \mathrm{l}$ ) and Anti-CD3 mAb (cat. no. AA3600, $100 \mu \mathrm{g} / 100 \mu \mathrm{l}$ ) were purchased from Abcam (Cambridge, MA, USA; both used at a 1:1000 dilution). Sterile PBS (10 mM, pH 7.4) was used as a buffer.

Preparation of the QDs-Nb36 complex. The quantum dot suspension droplets were dropped on a copper mesh, and left at room temperature until dry enough for transmission electron microscopy (TEM; model: H-7650; Hitachi, Ltd., Tokyo, Japan) analysis to confirm that the sample was qualified. Previously, Nb36 protein was obtained from a high quality dromedary camel immune library by phage display technology and expression in E. coli WK6 electrocompetent cells. After further purification, the Nb36 protein solution was obtained (10). First, Nb36 protein solution was dissolved in the PBS (to a final concentration of $1 \mathrm{mg} / \mathrm{ml}$ ). Subsequently, $50 \mu \mathrm{l}$ QDs solution and $100 \mu \mathrm{l}$ EDC solution $(0.8 \mathrm{mM})$ were mixed using a vortex mixer for $10 \mathrm{~min}$ at room temperature, then supplemented with $100 \mu \mathrm{l}$ NHS $(0.8 \mathrm{mM})$ and $300 \mu \mathrm{l}$ Nbs $(1 \mathrm{mg} / \mathrm{ml})$. The mixture was co-cultured for $1 \mathrm{~h}$ with gentle agitation at room temperature. Finally, unconjugated Nb36s were discarded. The conjugated QDs-Nb36 complexes were collected by centrifugation $(1,000 \mathrm{x} \mathrm{g}, 20 \mathrm{~min})$ at room temperature. Subsequently, the complexes were resuspended in PBS containing $1 \%(\mathrm{~m} / \mathrm{v})$ bovine serum albumin (BSA; cat. no. A7030; Sigma-Aldrich; Merck KGaA).

In vitro specificity of $Q D s-N b 36$ for polyhydroxyalkanoates (PHA)-stimulated human T cells. The peripheral blood mononuclear cells were isolated and cultured in RPMI-1640 medium (cat. no. 31800-021; Gibco; Thermo Fisher Scientific, Inc.) containing $10 \%$ fetal bovine serum (FBS; cat. no. 10099-141; Gibco; Thermo Fisher Scientific, Inc.) at $37^{\circ} \mathrm{C}$ for $2 \mathrm{~h}$. Subsequently, the adherent cells were removed and T cells were isolated using nylon-wool. PHA $(10 \mu \mathrm{g} / \mathrm{ml}$; cat. no. L8754; Sigma-Aldrich; Merck KGaA)-stimulated $\mathrm{T}$ cells $\left(1 \times 10^{6}\right)$ were incubated with $2 \%$ BSA solution for $30 \mathrm{~min}$ at $24^{\circ} \mathrm{C}$ with agitation to avoid non-specific binding. PHA is a phytohemagglutinin-specific polyclonal stimulator that stimulates human $\mathrm{T}$ cell proliferation through CD3-TCR on the cell membrane. A total of $4 \times 10^{5} \mathrm{~T}$ cells were resuspended in $300 \mu \mathrm{l}$ PBS for each sample. These samples were incubated with QDs-Nb36 in RPMI-1640 medium at $4{ }^{\circ} \mathrm{C}$ for $30 \mathrm{~min}$, followed by washing, suspension in PBS $(300 \mu \mathrm{l})$ and analysed using a Beckman Coulter flow cytometer with $\sim 10,000$ cells (Beckman Coulter, Inc., Brea, CA, USA). The fluorescence signal was detected on the fluorescein isothiocyanate channel. In the control groups, an equivalent dose of anti-CTLA-4 mAb and QDs were administered. Flowjo v10.07 software (FlowJo LLC, Ashland, OR, USA) was utilized for statistical analysis.

The PHA-stimulated T cells were washed with PBS three times and then fixed with $4 \%$ polyoxymethylene for 15 min at room temperature. After washing with PBS, the cell samples were incubated with QDs-Nb36 (100 nM), anti CTLA-4 mAb $(5 \mu \mathrm{l})$ and QDs (100 $\mathrm{nM})$ in binding buffer at $4^{\circ} \mathrm{C}$ for $50 \mathrm{~min}$ and washed with PBS three times. Subsequently, the samples were stained with DAPI $(1 \mu \mathrm{g} / \mathrm{ml})$ 


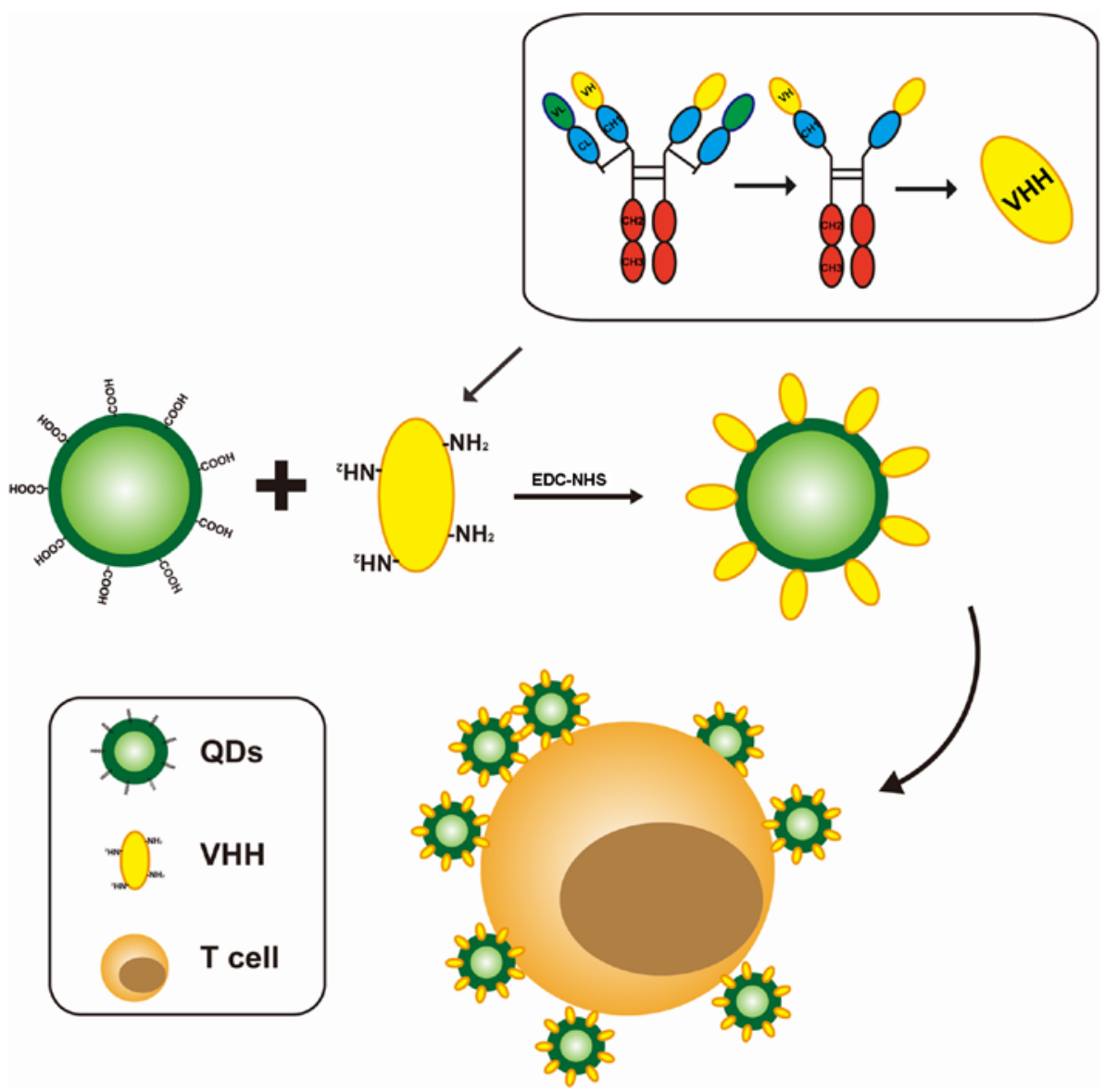

Figure 1. Schematic illustration of experimental principle: Highly sensitive detection of cytotoxic T-lymphocyte antigen-4-positive T cells using a QD-conjugated $\mathrm{Nb}$ (Nb36). CH, heavy chain constant domain; CL, light chain constant domain; EDC-NHS, 1-ethyl-3-(3-dimethylaminopropyl) carbodiimide hydrochloride N-hydroxysulfosuccinimide sodium salt; Nb, nanobody; QDs, quantum dots; VH, heavy chain variable domain; VHH, heavy chain; VL, light chain variable domain.

for $5 \mathrm{~min}$ at $4^{\circ} \mathrm{C}$. Images were captured using confocal microscopy (magnification, $\mathrm{x} 400$ ).

In vitro specificity of $Q D$ s-Nb36 for tumor-infiltrated $C T L A-4^{+}$ T cells. The tumor tissues and adjacent mucosa were immersed in optimal cutting temperature compound and processed into frozen sections $\left(-20^{\circ} \mathrm{C}\right)$. QDs-Nb36, anti CTLA-4 mAb and QDs were used for immunofluorescent staining. DAPI was used for nuclear staining. The frozen sections were observed using a confocal microscope (FV1000; Olympus Corporation, Japan; magnification, 400x). After the sample tissues were cut up, collagenase II (Sigma-Aldrich; Merck KGaA) was added for digestion. The tumor-infiltrated $\mathrm{T}$ cells isolated from tumor tissues and adjacent mucosa were processed using the anti-human CD3 monoclonal antibody immunomagnetic bead kit (cat. no. BMCD3; BioMag Corp., Wu Xi, China), as described previously $(17,18)$, then analyzed by flow cytometry. The specific antibodies used to detect the expression of CD3 and CTLA-4 were as follows: Anti-human CD3 mAb and QDs-Nb36 (100 nM), anti-CTLA-4 mAb or QDs (100 nM), incubated at $4^{\circ} \mathrm{C}$ for $30 \mathrm{~min}$. Data were analyzed using FlowJo v10.0.7 software.

In vitro toxicity of $Q D s-N b 36$. Toxicity of QDs-Nb36 against 293 cells was determined by MTT assay. The 293 cells $\left(2 \times 10^{5}\right)$ were cultured in RPMI-1640 medium containing 10\% FBS, in a 96-well plate overnight in a cell incubator $\left(5 \% \mathrm{CO}_{2}\right)$. Subsequently, the samples were treated with QDs-Nb36 $(10,20,30,40$ and $50 \mathrm{nM})$ for 24 or $48 \mathrm{~h}$. In the control group, an equivalent volume of PBS was administered. A total of $10 \mu \mathrm{l}$ MTT $(5 \mathrm{mg} / \mathrm{ml})$ was added to each well, and the plates were incubated at room temperature for $4 \mathrm{~h}$ in the dark. Subsequently, the medium was discarded, 150- $\mu$ l ethanol/dimethyl sulfoxide was added to each well and the cells were further incubated for $10 \mathrm{~min}$. The optical density of the samples was analyzed using an ELISA microplate reader (Thermo Fisher Scientific, Inc.) at a wavelength of $570 \mathrm{~nm}$.

In vivo toxicity of $Q D s-N b 36$. The mice received an intravenous injection of QDs-Nb36 $(15 \mathrm{mg} / \mathrm{kg})$ or PBS through the tail vein, once a week for 4 weeks. Alterations in the biological behavior and weight of the mice were monitored daily. A total of 7 days after treatment, serum was collected from the mice for biochemical examination with an AU5800 Clinical Chemistry Analyzer (Beckman Coulter, Inc.) The alanine aminotransferase, aspartate transaminase, serum creatinine and blood urea nitrogen levels were measured to assess liver and kidney function in mice. Subsequently, the animals were sacrificed. The primary organs, including spleen, kidney, heart, lung, liver and brain were immersed and preserved in 

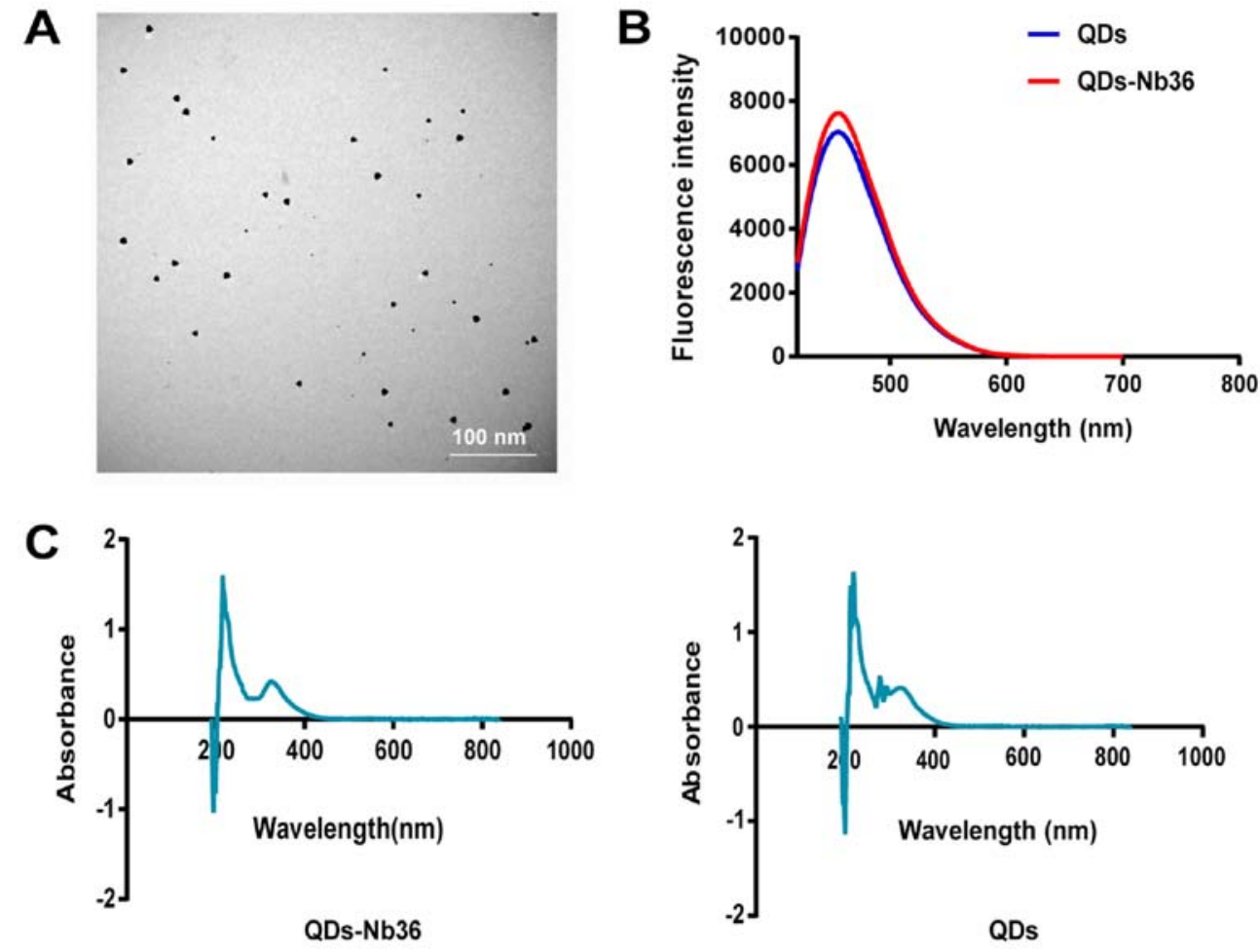

Figure 2. Characterization of the QDs-Nb36. (A) Transmission electron micrograph of QDs-Nb36. (B) Fluorescence emission spectra of QDs prior to and following modification. (C) Absorbance value of QDs prior to and following modification. QDs, quantum dots; QDs-Nb36, QDs-cytotoxic T-lymphocyte antigen-4-specific nanobody.

$10 \%$ formaldehyde solution at $4^{\circ} \mathrm{C}$ for $4 \mathrm{~h}$, dehydrated and embedded in paraffin. The paraffin-embedded sections $(4 \mu \mathrm{m})$ were stained with hematoxylin and eosin (HE) $(1 \mathrm{~min}$ each) at room temperature. Images were captured using a confocal microscope (magnification, 400x).

Statistical analysis. All experiments were repeated 3 times. Statistical analysis was performed using GraphPad Prism 6.02 software (GraphPad Software, Inc., La Jolla, CA, USA). Data were analyzed by Student's t-test or a Levene's test followed by a one-way analysis of variance and Newman-Keuls post-hoc test. $\mathrm{P}<0.05$ was considered to indicate a statistically significant difference. All data are expressed as the mean \pm standard deviation.

\section{Results}

Characterization of QDs-Nb36. The characteristics of QDs-Nb36 were analyzed. TEM data demonstrated that the dissemination of QDs-Nb36 was almost monodisperse (Fig. 2A). Their photoluminescence was measured using a fluorescence spectrophotometer (FL-7000; Perkin Elmer, Waltham, MA, USA). The fluorescence emission spectra peak of the QDs-Nb36 and QDs was $450 \mathrm{~nm}$ (Fig. 2B), and the absorbance peak was $350 \mathrm{~nm}$ (Fig. 2C).

Highly sensitive detection of CTLA- $4^{+}$T cells from human peripheral blood using QDs-Nb36. To characterize QDs-Nb36 binding to CTLA $-4^{+}$T cells, binding was analyzed using PHA-stimulated and normal T cells. The binding efficiency of QDs-Nb36 to target samples was evaluated by flow cytometry.
QDs-Nb36 and anti-CTLA-4 mAb specifically bound to CTLA- $4^{+} \mathrm{T}$ cells. A higher binding rate was identified for QDs-Nb36 compared with CTLA-4 mAb in PHA-stimulated cells and minimal binding of QDs was observed for all target cell lines (Fig. 3A and B). To obtain a more direct visualization of CTLA- $4^{+}$T cells using the QDs-Nb36 complex, confocal microscopy was performed to examine PHA-stimulated human $\mathrm{T}$ cells incubated with QDs-Nb36, anti-CTLA-4 mAb or QDs. The fluorescent images revealed that PHA-stimulated $\mathrm{T}$ cells exerted a significantly stronger fluorescence at the cell membrane in the presence of QDs-Nb36 compared with anti-CTLA-4 mAb, and no fluorescent signal was observed for QDs alone. When normal T cells were used as the sample, a small quantity of positive cells could be observed (Fig. 3C), suggesting that QDs-Nb36 complexes could be used in the detection of CTLA- $4^{+} \mathrm{T}$ cells with high sensitivity due to the specificity of Nb36 to CTLA-4.

Highly sensitive detection of tumor-infiltrated CTLA-4 ${ }^{+}$ $T$ cells using $Q D s-N b 36$. Next, immunofluorescent staining of the tumor tissues and adjacent mucosa was performed to investigate whether the fluorescence signal of infiltrated CTLA- $4^{+}$ $\mathrm{T}$ cells in the tumor tissues was significantly higher than that in adjacent mucosa for QDs-Nb36 or anti-CTLA-4 mAb. The number of positive cells detected using QDs-Nb36 was higher compared with anti-CTLA-4 mAb (Fig. 4A and B). Following isolation of tumor-infiltrated mononuclear leukocytes from the cancer tissue and adjacent mucosa, the expression levels of CTLA- 4 were measured by flow cytometry and the results were consistent with the findings of immunofluorescent staining (Fig. 4C and D). 
A

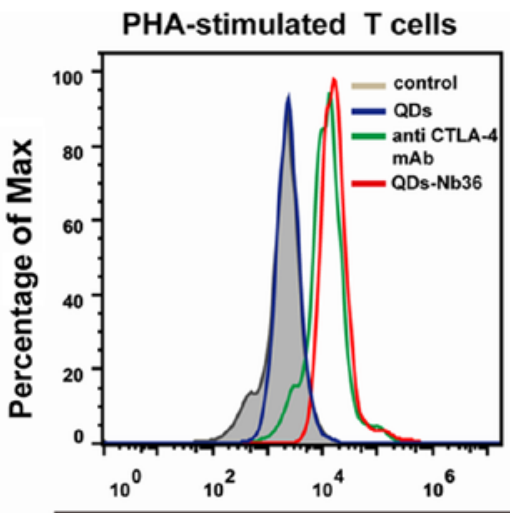

B

C

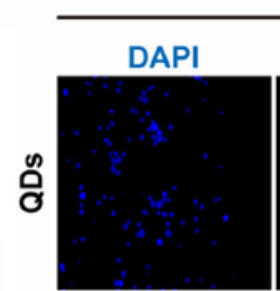

PHA-stimulated T cells
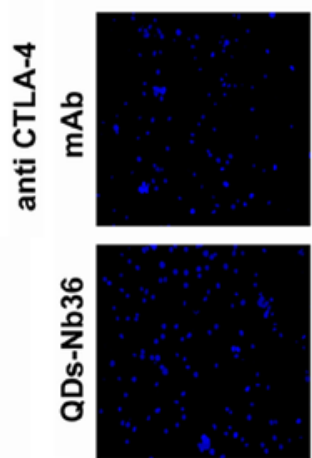

$\star \star \star$
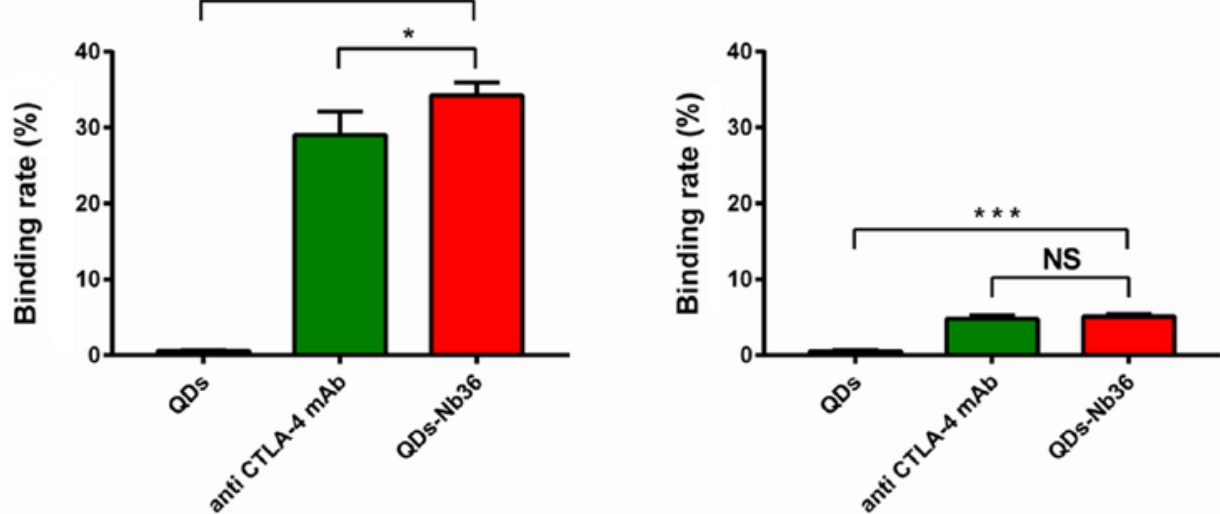

T cells

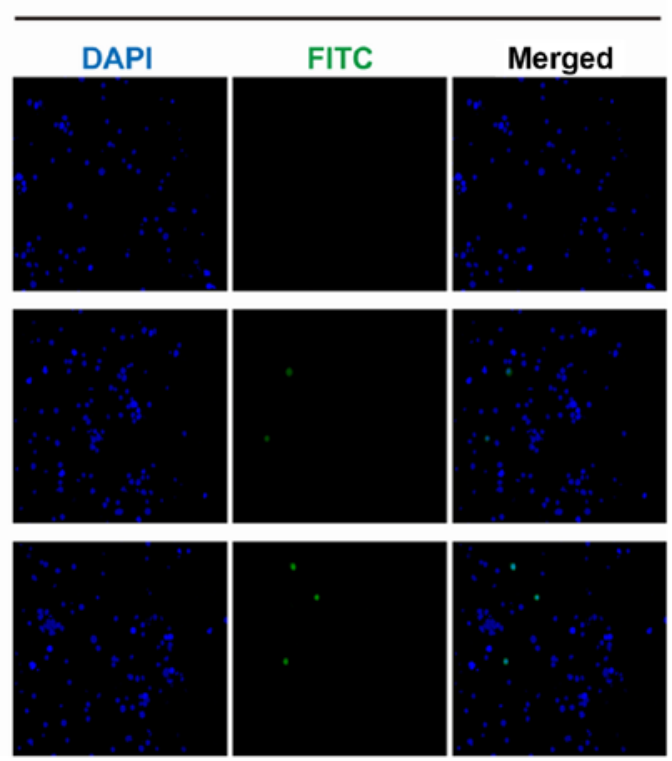

Figure 3. Specific detection of CTLA-4+ $\mathrm{T}$ cells with the QDs-Nb36 complex. (A) Flow cytometry of PHA-stimulated T cells and normal T cells following incubation with QDs, anti-CTLA-4 mAb or QDs-Nb36. (B) Quantitative analysis of PHA-stimulated and normal T cells. (C) Fluorescent micrographs of PHA-stimulated T cells and normal T cells following incubation with QDs, anti-CTLA-4 mAb or QDs-Nb36. Fluorescence was detected on the FITC channel, whereas DAPI-stained nuclei were detected on the blue channel. Magnification, $\mathrm{x} 400 .{ }^{~} \mathrm{P}<0.05,{ }^{* * *} \mathrm{P}<0.001$. CTLA-4, cytotoxic T-lymphocyte antigen-4; FITC, fluorescein isothiocyanate; mAb, monoclonal antibody; NS, not significant; PHA, polyhydroxyalkanoates; QDs, quantum dots; QDs-Nb36, QDs-CTLA-4-specific nanobody.

QDs-Nb36 exhibits non-toxicity in vitro and in vivo. An MTT assay confirmed that different concentrations of QDs-Nb36 exhibited no toxic effect on the normal cell line 293 after 24 and $48 \mathrm{~h}$ (Fig. 5A). The cytotoxicity of QDs-Nb36 in mice was subsequently analyzed. HE staining and serum biochemical examination were performed 1week after the last injection. As illustrated in Fig. 5C, no evident inflammation or necrosis was observed in the tissue sections. In addition, no significant differences in body weight gain and serum biochemistry of the treatment and control groups were 
A
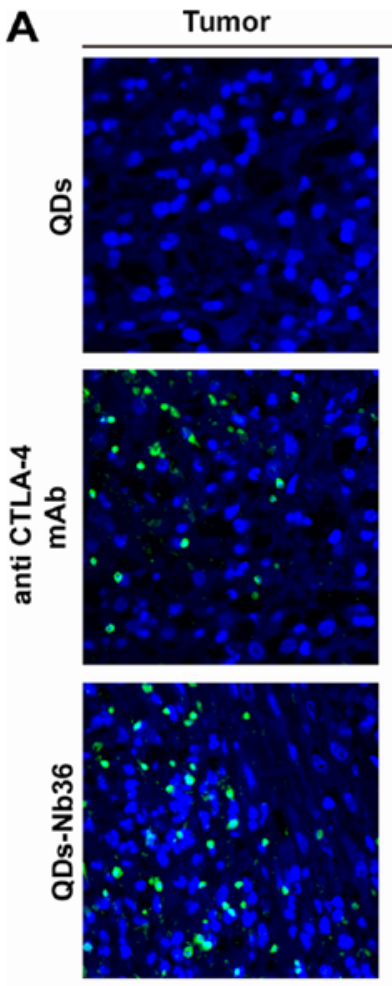

B

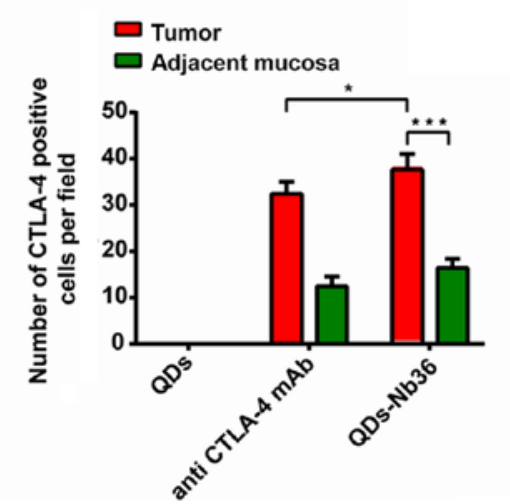

C

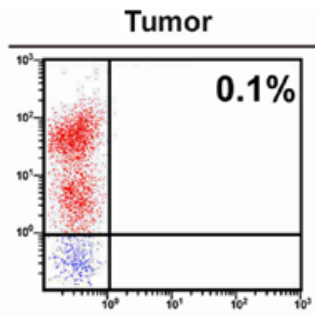

Adjacent mucosa
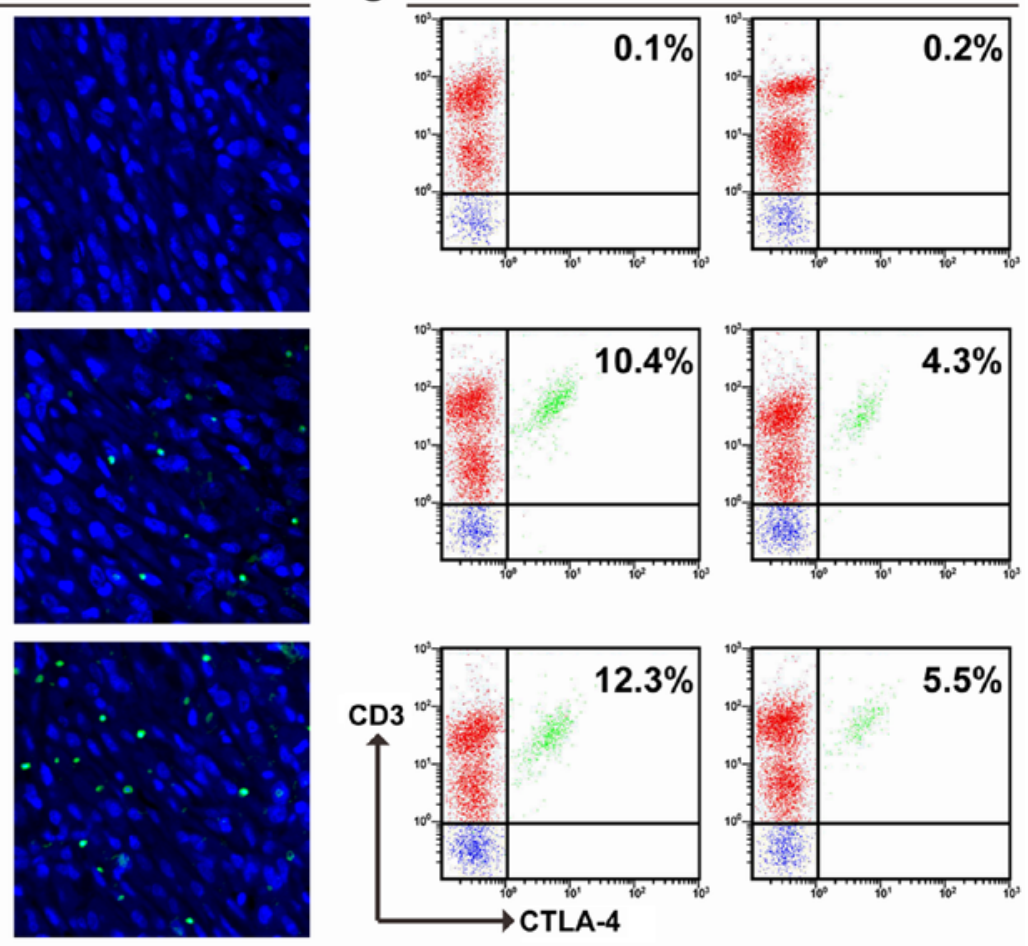

D

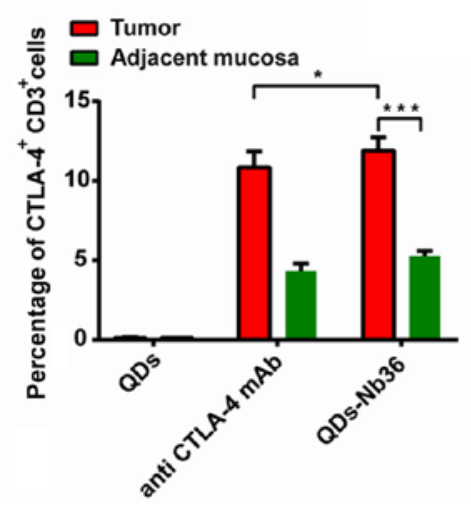

Figure 4. Specific detection of tumor-infiltrated CTLA-4+ T cells with the QDs-Nb36 complex. (A) Tumor tissues and adjacent mucosa were stained with QDs, anti CTLA-4 mAb or QDs-Nb36. Fluorescent images were examined in the fluorescein isothiocyanate channel, whereas DAPI-stained nuclei were examined in the blue channel. Magnification, x400. (B) Quantitative analysis of CTLA-4 ${ }^{+} \mathrm{T}$ cells per field. (C) Flow cytometry of tumor-infiltrated CTLA-4 ${ }^{+}$T cells isolated from tumor tissues and adjacent mucosa following incubation with anti-human CD3 mAb and QDs, anti-CTLA-4 mAb or QDs-Nb36. (D) Quantitative analysis of CTLA- $4^{+}$and CD3 ${ }^{+}$cells. ${ }^{*} \mathrm{P}<0.05,{ }^{* * *} \mathrm{P}<0.001$. CD3, cluster of differentiation 3; CTLA-4, cytotoxic T-lymphocyte antigen-4; QDs, quantum dots; QDs-Nb36, QDs-CTLA-4-specific nanobody.

identified (Fig. 5B and Table I). These results confirmed that QDs-Nb36 injection caused almost no cytotoxicity in mice.

\section{Discussion}

Nanoparticle probes based on graphene, liposome, silicon and the rare earth ions have been widely used in biomolecular imaging and diagnosis; however, the majority of these probes rely on complex synthesis methods and cannot provide direct fluorescence signals (19-22). Due to their specific optical and electronic features, semiconductor QDs serve as novel nanoparticle probes in bioimaging and bio-diagnostics (23). Nevertheless, the QD complexes retain certain limitations. It is of great significance to reduce the size of the complexes to lower steric hindrance and prevent the absorption of non-specific proteins (24). Heavy-chain antibodies (HCAbs) are unique in that they lack alight chain and are present in the serum of alpacas and llamas (25). In $\mathrm{HCAbs}$, the antigen-binding fragments consist of one variable domain. A type of antibody, termed Nbs, may be obtained by cloning the genes encoding this variable domain (26). It is the smallest antigen-binding antibody fragment compared with conventional antibodies, with a molecular weight of $\sim 15 \mathrm{kDa}, 4.8 \mathrm{~nm}$ length and $2.2 \mathrm{~nm}$ diameter. Due to their small molecular mass, Nbs may diffuse more efficiently throughout solid tumors. Additionally, Nbs possess several inherent characteristics, including high physiochemical stability, high binding specificity, high affinity $\left(K_{D}<10 \mathrm{nM}\right)$ and rapid clearance in the bloodstream, which are applicable in quantitative bioassays (27). 
Table I. Serum biochemical examination of mice in the QDs-Nb36 group compared with the PBS group.

\begin{tabular}{llllr}
\hline Group & ALT, U/ml & AST, U/ml & SCr, $\mu \mathrm{mol} / 1$ & BUN, $\mu \mathrm{mol} / 1$ \\
\hline PBS & $20.66 \pm 1.73$ & $41.27 \pm 2.94$ & $21.73 \pm 3.75$ & $11.51 \pm 1.36$ \\
QDs-Nb36 & $18.34 \pm 1.68$ & $45.21 \pm 4.31$ & $20.96 \pm 3.41$ & $12.97 \pm 1.55$ \\
\hline
\end{tabular}

ALT, alanine aminotransferase; AST, aspartate transaminase; BUN, blood urea nitrogen; QDs-Nb36, quantum dots- cytotoxic T-lymphocyte antigen-4-specific nanobody; SCr, serum creatinine.

A

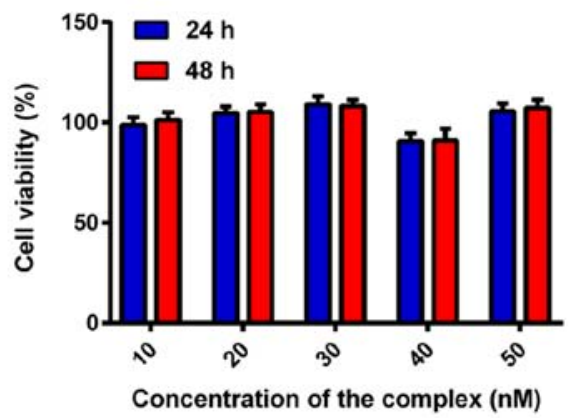

C
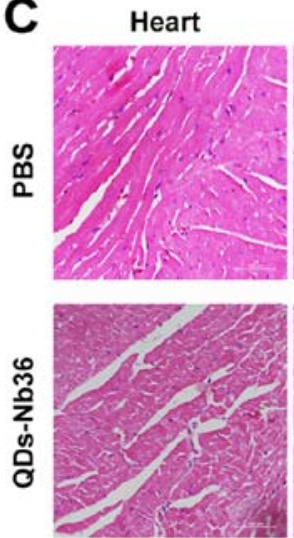

Liver
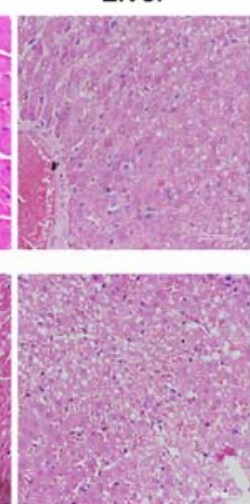
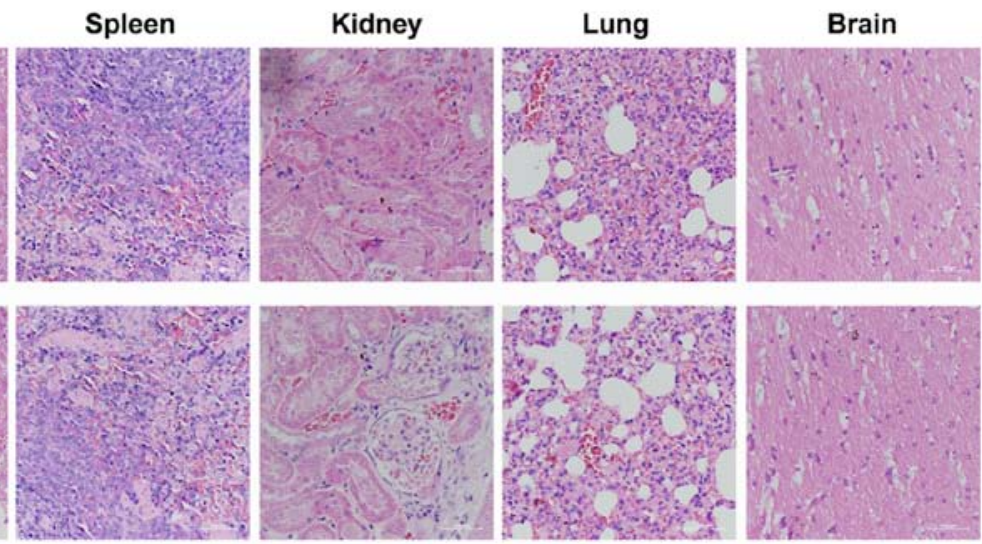

Figure 5. Toxicity of QDs-Nb36. (A) Cells were incubated with various concentrations (10, 20, 30, 40 and $50 \mathrm{nM}$ ) of QDs-Nb36 complex, and cell viability was measured at 24 and $48 \mathrm{~h}$. (B) Weight of mice following QDs-Nb36 injection. (C) C57BL/6 mice were injected with QDs-Nb36 or PBS. Sections from primary organs were stained with hematoxylin-eosin and images were captured using confocal microscopy. Magnification, x400. NS, not significant; QDs-Nb36, quantum dots-cytotoxic T-lymphocyte antigen-4-specific nanobody.

The synthesis of the CTLA-4-specificNbs and QDs complex is a simple and efficient strategy for detecting CTLA- $4^{+}$T cells. Anti-CTLA-4 Nbs specifically bind to CTLA- $4^{+}$T cells, whereas QDs provide a sensitive fluorescent signal for accurate detection. Anamide bond between a carboxylic acid group on QDs and an amine group on NBs maybe used to construct the QDs-Nb36 complex. In the present study, in vitro imaging demonstrated that the fluorescent intensity in PHA-stimulated human $\mathrm{T}$ cells and tumor sections was significantly higher for the QDs-Nb36 complex compared with that of the anti-CTLA-4 mAb. In addition, significant differences in the binding rates between the two groups were also identified in tumor-infiltrated $\mathrm{T}$ cells, which demonstrated the specific binding of the QDs-Nb36 complex to CTLA- $4^{+}$ cells in vitro. These findings suggested that the efficiency of QDs-Nb36 detection was higher than that of anti-CTLA-4 $\mathrm{mAb}$. The method of QDs-Nb36 synthesis may increase the binding of Nbs and QDs to enhance specificity and fluorescence intensity.

The toxicity of QDs-Nb36 in vitro and in vivo was assessed by MTT assay, HE staining and serum biochemical examination. QDs-Nb36 yielded no toxicity to normal human cells and the primary organs of mice 4 weeks post-injection. In addition, it exerted no effect on the body weight of mice, indicating that the QDs-Nb36 complex is not only a safe in vitro detection tool, but has the potential for in vivo assessment or treatment of tumor suppressor $\mathrm{T}$ cells.

In conclusion, a novel complex was developed to detect CTLA- $4^{+} \mathrm{T}$ cells based on a CTLA-4-specific Nb and QDs. The specificity of the $\mathrm{Nb}$ for CTLA- $4^{+}$human $\mathrm{T}$ cells coupled with QDs, which provided a strong fluorescent signal, led to high specificity and sensitivity in the detection of the CTLA $-4^{+}$ T-cell ratio. The fluorescent intensity of QDs was significantly higher compared with a mAb. The complex was superior to mAbs in monitoring CTLA $-4^{+}$cell number. Furthermore, 
this method may be used for the detection of other biological targets using Nbs specific to other targets.

\section{Acknowledgements}

Not applicable.

\section{Funding}

The present study was supported, in part, by grants from The National Natural Scientific Foundation of China (grant no. 81773254); Programs for Changjiang Scholars and Innovative Research Team in University (grant no.IRT_15R13); International Cooperation Project of the Ministry of Science and Technology of China (grant no. 2015DFA31320); Project for Innovative Research Team in Guangxi Natural Science Foundation (grant no. 2015GXNSFFA139001); and The Project for International Nanobody Research Center of Guangxi (grant no. GuiKe-AD17195001).

\section{Availability of data and materials}

The datasets used and/or analyzed during the present study are available from the corresponding author on reasonable request.

\section{Authors' contributions}

WW, XH, XY, AL, ZT, FM and SY performed the experiments. XL performed the statistical analysis and wrote the manuscript.

\section{Ethics approval and consent to participate}

The present animal study was approved by Guangxi Medical University. The study procedures were approved by The Animal Ethics Committee of Guangxi Medical University (Guangxi, China).

\section{Patient consent for publication}

Not applicable.

\section{Competing interests}

The authors declare that they have no competing interests.

\section{References}

1. Lenschow DJ, Walunas TL and Bluestone JA: CD28/B7 system of T cell costimulation. Annu Rev Immunol 14: 233-258, 1996.

2. Teft WA, Kirchhof MG and Madrenas J: A molecular perspective of ctla-4 function. Annu Rev Immunol 24: 65-97, 2006.

3. Walker LS and Sansom DM: The emerging role of ctla4 as a cell-extrinsic regulator of $\mathrm{T}$ cell responses. Nat Rev Immunol 11: $852-863,2011$

4. Wakamatsu E, Mathis D and Benoist C: Convergent and divergent effects of costimulatory molecules in conventional and regulatory CD4 ${ }^{+}$T cells. Proc Natl Acad Sci USA 110: 1023-1028, 2013.

5. Krummel MF and Allison JP: CD28 and ctla-4 have opposing effects on the response of T cells to stimulation. J Exp Med 182: 459-465, 1995.

6. Ichihara F, Kono K, Takahashi A, Kawaida H, Sugai H and Fujii H: Increased populations of regulatory $\mathrm{T}$ cells in peripheral blood and tumor-infiltrating lymphocytes in patients with gastric and esophageal cancers. Clin Cancer Res 9: 4404-4408, 2003.
7. Aggarwal S, Sharma SC and N Das S: Dynamics of regulatory $\mathrm{T}$ cells (Tregs) in patients with oral squamous cell carcinoma. J SurgOncol 116: 1103-1113, 2017.

8. Benevides L, Cardoso CR, Tiezzi DG, Marana HR, Andrade JM and Silva JS: Enrichment of regulatory T cells in invasive breast tumor correlates with the upregulation of IL-17A expression and invasiveness of the tumor. Eur J Immunol 43: 1518-1528, 2013.

9. Steeland S, Vandenbroucke RE and Libert C: Nanobodies as therapeutics: Big opportunities for small antibodies. Drug Discov Today 21: 1076-1113, 2016.

10. Wan R, Liu A1, Hou X, Lai Z, Li J, Yang N, Tan J, Mo F, Hu Z, Yang $\mathrm{X}$, et al: Screening and antitumor effect of an anti-CTLA-4 nanobody. Oncol Rep 39: 511-518, 2018

11. Muyldermans S: Nanobodies: Natural single-domain antibodies. Annual Review of Biochemistry: 775-797, 2013.

12. Zhang M, Liu H, Chen L, Yan M, Ge L, Ge S and Yu J: A disposable electrochemiluminescence device for ultrasensitive monitoring of K562 leukemia cells based on aptamers and ZnO@ carbon quantum dots. Biosens Bioelectron 49: 79-85, 2013.

13. Yu Y, Duan S, He J, Liang W, Su J, Zhu J, Hu N, Zhao Y and Lu X: Highly sensitive detection of leukemia cells based on aptamer and quantum dots. Oncol Rep 36: 886-892, 2016.

14. Qu K, Wang J, Ren J and Qu X: Carbon dots prepared by hydrothermal treatment of dopamine as an effective fluorescent sensing platform for the label-free detection of iron (III) ions and dopamine. Chemistry 19: 7243-7249, 2013.

15. Yang ST, Wang X, Wang H, Lu F, Luo PG, Cao L, Meziani MJ, Liu JH, Liu Y, Chen M, et al: Carbon dots as nontoxic and high-performance fluorescence imaging agents. J Phys Chem C Nanomater Interfaces 113: 18110-18114, 2009.

16. Xing Y and Rao J: Quantum dot bioconjugates for in vitro diagnostics \& in vivo imaging. Cancer Biomark 4: 307-319, 2008.

17. Peter PA, Durflinger KH, Wunderlich JR, Rosenberg SA, and Dudley ME: Enrichment of CD8+ cells from melanoma tumor-infiltrating lymphocyte cultures reveals tumor reactivity for use in adoptive cell therapy. J Immunother 33: 547-556, 2010.

18. Tran KQ, Zhou J, Durflinger KH, Langhan MM, Shelton TE, Wunderlich JR, Robbins PF, Rosenberg SA and Dudley ME: Minimally cultured tumor-infiltrating lymphocytes display optimal characteristics for adoptive cell therapy. J Immunother 31: 742-751, 2008.

19. Zhang L, Wang Z, Lu Z, Xia K, Deng Y, Li S, Zhang C, Huang Y and He N: Synthesis of LiYF4:Yb, Erupconversion nanoparticles and its fluorescence properties. J NanosciNanotechnol 14: 4710-4713, 2014

20. Heo NS, Lee SU, Rethinasabapathy M, Lee EZ, Cho HJ, Oh SY, Choe SR, Kim Y, Hong WG, Krishnan G, et al: Visible-light-driven dynamic cancer therapy and imaging using graphitic carbon nitride nanoparticles. Mater Sci Eng C Mater Biol Appl 90: 531-538, 2018.

21. Ji X, Wang H, Song B, Chu B and He Y: Silicon nanomaterials for biosensing and bioimaging analysis. Front Chem 6: 38, 2018.

22. Lamichhane N, Udayakumar TS, D'Souza WD, Simone CB II, Raghavan SR, Polf J and Mahmood J: Liposomes: Clinical applications and potential for image-guided drug delivery. Molecules 23: 288, 2018.

23. Alivisatos P: The use of nanocrystals in biological detection. Nat Biotechnol 22: 47-52, 2004.

24. Baker M: Nanotechnology imaging probes: Smaller and more stable. Nat Methods 7: 957-962, 2010.

25. Hamers-Casterman C, Atarhouch T, Muyldermans S, Robinson G, Hamers C, Songa EB, Bendahman N and Hamers R: Naturally occurring antibodies devoid of light chains. Nature 363: 446-448, 1993.

26. Deffar K, Shi H, Li L, Wang X and Zhu X: Nanobodies-the new concept in antibody engineering. Afr J Biotechnol 8: 2645-2652, 2009.

27. Hisada $H$, Tsutsumi $H$, Ishida $H$ and Hata $Y$ : High production of llama variable heavy-chain antibody fragment $(\mathrm{VHH})$ fused to various reader proteins by Aspergillusoryzae. Appl Microbiol Biotechnol 97: 761-766, 2013.

(i) $\ominus$ This work is licensed under a Creative Commons Attribution-NonCommercial-NoDerivatives 4.0 International (CC BY-NC-ND 4.0) License. 